The Journal of Nonlinear $\mathbf{S}_{\text {cience and }}$ Applications

http://www.tjnsa.com

\title{
STRONG CONVERGENCE THEOREMS FOR EQUILIBRIUM PROBLEMS AND FIXED POINT PROBLEMS OF STRICT PSEUDO-CONTRACTION MAPPINGS
}

\author{
LIANG CAI ZHAO ${ }^{1, *}$ AND SHIH-SEN CHANG ${ }^{1,2}$
}

\begin{abstract}
The purpose of this paper is to introduce an iterative scheme for finding a common element of the set of solutions of an equilibrium problem and the set of fixed points of a $k$-strict pseudo-contraction non-self mapping in Hilbert space. By the viscosity approximation algorithms, under suitable conditions, some strong convergence theorems for approximating to this common elements are proved. The results presented in the paper extend and improve some recent results of Marino and Xu [G.Marino,H.K.Xu, Weak and strong convergence theorems for $k$-strict pseudo-contractions in Hilbert spaces, J. Math. Anal. Appl. 329 (2007) 336-349], Zhou [H.Zhou, Convergence theorems of fixed Points for $k$-strict pseudo-contractions in Hilbert spaces, Nonlinear Anal. 69 (2008) 456-462], Takahashi and Takahashi [S. Takahashi, W. Takahashi, Viscosity approximation methods for equilibrium problems and fixed point problems in Hilbert spaces, J. Math. Anal. Appl. 331 (2007) 506515], Ceng,Homidan,etc [L. C. Ceng, S.A.Homidan, Q.H.Ansari, J. C. Yao, An iterative scheme for equilibrium problems and fixed point problems of strict pseudo-contraction mappings, J. Comput. Appl. Math. 223 (2009) 967-974].
\end{abstract}

\section{INTRODUCTION}

Throughout this paper, we assume that $H$ is a real Hilbert space with inner product $\langle\cdot, \cdot\rangle$ and norm $\|\cdot\| . C$ is a nonempty closed convex subset of $H$. Let $\phi: C \times C \rightarrow \mathbb{R}$ be a bifunction, where $\mathbb{R}$ is the set of real numbers. The equilibrium problem (for short, EP) is to find $x \in C$ such that

$$
\phi(x, y) \geq 0, \quad \forall y \in C .
$$

Date: Received: 24 February 2009. Revised 8 March 2009.

${ }^{*}$ Corresponding author.

2000 Mathematics Subject Classification. 47H05. 47H09. 47H10.

Key words and phrases. Equilibrium problem, strict pseudo-contraction mapping, fixed point, strong convergence theorem. 
The set of solutions of (1.1) is denoted by $E P(\phi)$. Given a mapping $T: C \rightarrow H$, let $\phi(x, y)=\langle T x, y-x\rangle$ for all $x, y \in C$. Then $x \in E P(\phi)$ if and only if $x \in C$ is a solution of the variational inequality $\langle T x, y-x\rangle \geq 0$ for all $y \in C$. In addition, there are several other problems, for example, the complementarity problem, fixed point problem and optimization problem, which can also be written in the form of an EP. In other words, the EP is an unifying model for several problems arising in physics, engineering, science, optimization, economics, etc. In the last two decades, many papers have appeared in the literature on the existence of solutions of EP; see, for example [1,8,9] and references therein. Some solution methods have been proposed to solve the $E P$; see, for example $[6,7,20,21]$ and references therein. Motivated by the work in [6,14,20], Takahashi and Takahashi [21] introduced an iterative scheme by the viscosity approximation method for finding a common element of the set of solutions of the $E P(1.1)$ and the set of the fixed points of a nonexpansive mapping in the setting of Hilbert spaces. They also studied the strong convergence of the sequences generated by their algorithm for a solution of the EP which is also a fixed point of a nonexpansive mapping defined on a closed convex subset of a Hilbert space.

Very recently, Ceng, Homidan, Ansari and Yao [4] introduced an iterative scheme for finding a common element of the set of solutions of the $E P(1.1)$ and the set of the fixed points of a $k$-strict pseudo-contraction self-mapping in the setting of real Hilbert spaces. They proved some weak and strong convergence theorems of the sequences generated by their proposed scheme.

Recall that a mapping $f: H \rightarrow H$ is said to be contractive if there exists a constant $\alpha \in(0,1)$ such that for all $x, y \in H$

$$
\|f(x)-f(y)\| \leq \alpha\|x-y\| .
$$

Let $A$ be a strongly positive bounded linear operator on $H$, that is, there exists a constant $\widetilde{\gamma}>0$ such that

$$
\langle A x, x\rangle \geq \widetilde{\gamma}\|x\|^{2}, \quad \forall x \in H .
$$

A mapping $T: H \rightarrow H$ is called nonexpansive, if such that

$$
\|T x-T y\| \leq\|x-y\|, \quad \forall x, y \in H .
$$

We denote by $F(T)$ the set of all fixed points of $T$, that is $F(T)=\{x \in H: T x=$ $x\}$. The mapping $T: C \rightarrow H$ is called a $k$-strict pseudo-contraction if there exists a constant $k \in[0,1)$ such that

$$
\|T x-T y\|^{2} \leq\|x-y\|^{2}+k\|(I-T) x-(I-T) y\|^{2}
$$

for all $x, y \in C$. When $k=0, T$ is said to be nonexpansive, and it is said to be pseudo-contractive if $k=1$. $T$ is said to be strongly pseudo-contractive if there exists a positive constant $\lambda \in(0,1)$ such that $T-\lambda I$ is pseudo-contractive. Cleary, the class of $k$-strict pseudo-contractions falls into the one between classes of nonexpansive mappings and pseudo-contractions. We remark also that the class of strongly pseudo-contractive mappings is independent of the class of $k$-strict pseudo-contractions (see $[2,3])$. 
It is very clear that,in a real Hilbert space $H,(1.2)$ is equivalent to

$$
\langle T x-T y, x-y\rangle \leq\|x-y\|^{2}-\frac{1-k}{2}\|(x-T x)-(y-T y)\|^{2}
$$

for all $x, y \in C$.

Recall that the normal Mann's iterative algorithm was introduced by Mann[12] in 1953. Since then,construction of fixed points for nonexpansive mappings and $k$-strict pseudo-contractions via the normal Mann's iterative algorithm has been extensively investigated by many authors (see, e.g.,[2,3,12,13,15,22]). Reich [17] showed that the conclusion also holds good in the setting of uniformly convex Banach spaces with a Fréchet differentiable norm.It is well known that Reich's result is one of the fundamental convergence results.Recently, Marino and Xu [13] extended Reich's result [17] to strict pseudo-contraction mappings in the setting of Hilbert spaces.

Very recently, Zhou [25] modified normal Mann's iterative process for non-self $k$-strict pseudo-contractions to have strong convergence in Hilbert spaces.

Motivated and inspired by Ceng, Homidan, Ansari and Yao [4], Marino and $\mathrm{Xu}$ [13], Takahashi and Takahashi [21], Zhou [25], the purpose of this paper is to introduce an iterative scheme for finding a common element of the set of solutions of equilibrium problem (1.1) and the set of fixed points of a $k$-strict pseudocontraction non-self mapping in Hilbert space. By the viscosity approximation algorithms, under suitable conditions, some strong convergence theorems for approximating to this common elements are proved. The results presented in the paper extend and improve some recent results of Ceng, Homidan, Ansari and Yao [4], Kim and Xu [11], Marino and Xu [13], Moudafi [14], Takahashi and Takahashi [21], Wittmann [23], Zhou [25].

\section{PRELIMINARIES}

In the sequel, we use $x_{n} \rightarrow x$ and $x_{n} \rightarrow x$ to denote the weak convergence and strong convergence of the sequence $\left\{x_{n}\right\}$ in $H$, respectively. Let $H$ be a real Hilbert space, $C$ be a nonempty closed convex subset of $H$. For any $x \in H$, there exists a unique nearest point in $C$, denoted by $P_{C}(x)$, such that

$$
\left\|x-P_{C} x\right\| \leq\|x-y\|, \quad \forall y \in C .
$$

Such a mapping $P_{C}$ from $H$ onto $C$ is called the metric projection.

Remark 1 It is wellknown that the metric projection $P_{C}$ has the following properties:

(1) $P_{C}$ is firmly nonexpansive. i.e.,

$$
\left\|P_{C} x-P_{C} y\right\|^{2} \leq\left\langle P_{C} x-P_{C} y, x-y\right\rangle, \quad \forall x, y \in H,
$$

(2) For each $x \in H$,

$$
z=P_{C}(x) \Leftrightarrow\langle x-z, z-y\rangle \geq 0, \quad \forall y \in C .
$$

A spave $X$ is said to satisfy the Opial condition if for each sequence $\left\{x_{n}\right\}$ in $X$ which converges weakly to a point $x \in X$, we have

$$
\liminf _{n \rightarrow \infty}\left\|x_{n}-x\right\|<\liminf _{n \rightarrow \infty}\left\|x_{n}-y\right\|, \quad \forall y \in X, y \neq x .
$$


Lemma 2.1. ([13]) Let $H$ be a real Hilbert space. There hold the following identities:

$$
\|t x+(1-t) y\|^{2}=t\|x\|^{2}+(1-t)\|y\|^{2}-t(1-t)\|x-y\|^{2}, \forall t \in[0,1],
$$

for all $x, y \in H$.

Lemma 2.2. ([19]) Let $\left\{x_{n}\right\}$ and $\left\{z_{n}\right\}$ be bounded sequences in a Banach space $E$ and let $\left\{\beta_{n}\right\}$ be a sequence in $[0,1]$ with $0<\liminf _{n \rightarrow \infty} \beta_{n} \leq \limsup _{n \rightarrow \infty} \beta_{n}<1$. Suppose that

$$
x_{n+1}=\left(1-\beta_{n}\right) z_{n}+\beta_{n} x_{n},
$$

for all integers $n \geq 1$ and

$$
\limsup _{n \rightarrow \infty}\left(\left\|z_{n+1}-z_{n}\right\|-\left\|x_{n+1}-x_{n}\right\|\right) \leq 0
$$

Then, $\lim _{n \rightarrow \infty}\left\|z_{n}-x_{n}\right\|=0$.

Lemma 2.3. ([24]) Assume $\left\{a_{n}\right\}$ is a sequence of nonnegative real numbers such that

$$
a_{n+1} \leq\left(1-\varrho_{n}\right) a_{n}+\epsilon_{n}, \quad \forall n \geq n_{0},
$$

where $n_{0}$ is some nonnegative integer, $\left\{\varrho_{n}\right\}$ is a sequence in $(0,1)$ and $\left\{\epsilon_{n}\right\}$ is a sequence such that

(1) $\sum_{n=1}^{\infty} \varrho_{n}=\infty$;

(2) $\limsup _{n \rightarrow \infty} \epsilon_{n} / \varrho_{n} \leq 0$ or $\sum_{n=1}^{\infty}\left|\epsilon_{n}\right|<\infty$.

Then $\lim _{n \rightarrow \infty} a_{n}=0$.

Lemma 2.4. ([13,25]) If $T$ is a $k$-strict pseudo-contraction on closed convex subset $C$ of a real Hilbert space $H$, then the fixed point set $F(T)$ is closed convex so that the projection $P_{F(T)}$ is well defined.

Lemma 2.5. ([3]) Let $T: C \rightarrow H$ be a $k$-strict pseudo-contraction. Define $S: C \rightarrow H$ by $S x=\lambda x+(1-\lambda) T x$ for each $x \in C$. Then, as $\lambda \in[k, 1), S$ is a nonexpansive mapping such that $F(S)=F(T)$.

Lemma 2.6. ([5]) let $E$ be a real Banach space, $J: E \rightarrow 2^{E^{*}}$ be the normalized duality mapping, Then for any $x, y \in E$. the following conclusion holds:

$$
\|x+y\|^{2} \leq\|x\|^{2}+2\langle y, j(x+y)\rangle, \quad \forall j(x+y) \in J(x+y)
$$

Especially, if $E=H$ is a real Hilbert space, then

$$
\|x+y\|^{2} \leq\|x\|^{2}+2\langle y, x+y\rangle, \quad \forall x, y \in H .
$$

For solving the equilibrium problem, we assume that the bifunction $\phi: C \times C \rightarrow$ $\mathbb{R}$ satisfies the following conditions:

(A1) $\phi(x, x)=0, \forall x \in C$,

(A2) $\phi$ is monotone, that is, $\phi(x, y)+\phi(y, x) \leq 0, \forall x, y \in C$,

(A3) For all $x, y, z \in C$,

$$
\limsup _{t \downarrow 0} \phi(t z+(1-t) x, y) \leq \phi(x, y), \forall x, y, z \in C,
$$


(A4) For all $x \in C$, the function $y \mapsto \phi(x, y)$ is convex and lower semicontinuous.

Lemma 2.7. ([6,21]) Let $C$ be a nonempty closed convex subset of a $H$ and let $\phi: C \times C \rightarrow \mathbb{R}$ be a bifunction satisfying $(A 1)-(A 4)$. Let $r>0$ and $x \in H$. Then, there exists $z \in C$ such that

$$
\phi(z, y)+\frac{1}{r}\langle y-z, z-x\rangle \geq 0, \quad \forall y \in C .
$$

Lemma 2.8. ([6]) Assume that $\phi: C \times C \rightarrow \mathbb{R}$ satisfying $(A 1)-(A 4)$. For $r>0$ and $x \in H$, define a mapping $T_{r}: H \rightarrow C$ as follows:

$$
T_{r}(x)=\left\{z \in C: \phi(z, y)+\frac{1}{r}\langle y-z, z-x\rangle \geq 0, \forall y \in C\right\} .
$$

Then,

(1) $T_{r}$ is single-valued,

(2) $T_{r}$ is firmly nonexpansive, that is, $\forall x, y \in H$,

$$
\left\|T_{r} x-T_{r} y\right\|^{2} \leq\left\langle T_{r} x-T_{r} y, x-y\right\rangle,
$$

(3) $F\left(T_{r}\right)=E P(\phi)$,

(4) $\operatorname{EP}(\phi)$ is nonempty, closed and convex.

\section{MAIN RESULTS}

Theorem 3.1. Let $H$ be a real Hilbert space, $C$ be a nonempty closed convex subset of $H$. Let $\phi: C \times C \rightarrow \mathbb{R}$ be a bifunction satisfying $(A 1)-(A 4)$. Let $A$ be a strongly positive linear bounded operator on $H$ with coefficient $\widetilde{\gamma}>0$ such that $0<\gamma<\tilde{\gamma} / \alpha$. $T: C \rightarrow H$ be a $k$-strictly pseudo-contractive nonself-mapping such that $F(T) \cap E P(\phi) \neq \emptyset$, and $f: H \rightarrow H$ be a contractive mapping with a contractive constant $\alpha \in(0,1)$. For any given $x_{1} \in H$, let $\left\{x_{n}\right\}$ and $\left\{u_{n}\right\}$ be the iterative sequence defined by

$$
\begin{cases}\phi\left(u_{n}, y\right)+\frac{1}{r_{n}}\left\langle y-u_{n}, u_{n}-x_{n}\right\rangle \geq 0, & \forall y \in C, \\ y_{n}=\delta_{n} u_{n}+\left(1-\delta_{n}\right) T u_{n}, & \forall n \geq 1 . \\ x_{n+1}=\alpha_{n} \gamma f\left(x_{n}\right)+\beta_{n} x_{n}+\left(\left(1-\beta_{n}\right) I-\alpha_{n} A\right) y_{n}, & \forall n . \beta\end{cases}
$$

where $\left\{\alpha_{n}\right\},\left\{\beta_{n}\right\},\left\{\delta_{n}\right\}$ are three sequences in $[0,1]$ and $r_{n} \subset(0, \infty)$. If the following conditions are satisfied:

(i) $\lim _{n \rightarrow \infty} \alpha_{n}=0 ; \quad \sum_{n=1}^{\infty} \alpha_{n}=\infty$,

(ii) $k \leq \delta_{n} \leq \lambda<1$ for all $n \geq 1$ and $\sum_{n=1}^{\infty}\left|\delta_{n+1}-\delta_{n}\right|<\infty$,

(iii) $0<\liminf _{n \rightarrow \infty} \beta_{n} \leq \limsup _{n \rightarrow \infty} \beta_{n}<1$,

(iv) $\liminf _{n \rightarrow \infty} r_{n}>0, \lim _{n \rightarrow \infty}\left|r_{n+1}-r_{n}\right|=0$.

Then $\left\{x_{n}\right\}$ and $\left\{u_{n}\right\}$ cnoverge strongly to $p \in F(T) \cap E P(\phi)$, where $p=P_{F(T) \cap E P(\phi)}(I-$ $A+\gamma f)(p)$. 
Proof. We divide the proof of Theorem 3.1 into seven steps:

(I) First prove that there exists $x^{*} \in C$, such that $x^{*}=P_{F(T) \cap E P(\phi)}(I-A+$ $\gamma f)\left(x^{*}\right)$.

Note that for the control conditions (i) and (iii), we may assume, without loss of generality, that $\alpha_{n} \leq\left(1-\beta_{n}\right)\|A\|^{-1}$. Since $A$ is linear bounded self-adjoint operator on $H$, then

$$
\|A\|=\sup \{|\langle A u, u\rangle|: u \in H,\|u\|=1\} .
$$

Observe that

$$
\begin{aligned}
\left\langle\left(\left(1-\beta_{n}\right) I-\alpha_{n} A\right) u, u\right\rangle & =1-\beta_{n}-\alpha_{n}\langle A u, u\rangle \\
& \geq 1-\beta_{n}-\alpha_{n}\|A\| \\
& \geq 0
\end{aligned}
$$

that is to say $\left(1-\beta_{n}\right) I-\alpha_{n} A$ is positive. It follows that

$$
\begin{aligned}
\left\|\left(1-\beta_{n}\right) I-\alpha_{n} A\right\| & =\sup \left\{\left\langle\left(\left(1-\beta_{n}\right) I-\alpha_{n} A\right) u, u\right\rangle: u \in H,\|u\|=1\right\} \\
& =\sup \left\{1-\beta_{n}-\alpha_{n}\langle A u, u\rangle: u \in H,\|u\|=1\right\} \\
& \leq 1-\beta_{n}-\alpha_{n} \widetilde{\gamma} .
\end{aligned}
$$

Since $f$ is a contraction with coefficient $\alpha \in(0,1)$. Then, we have

$$
\begin{aligned}
\| P_{F(T) \cap E P(\phi)}(I-A & +\gamma f)(x)-P_{F(T) \cap E P(\phi)}(I-A+\gamma f)(y) \| \\
& \leq\|(I-A+\gamma f)(x)-(I-A+\gamma f)(y)\| \\
& \leq\|I-A\|\|x-y\|+\gamma\|f(x)-f(y)\| \\
& \leq(1-\widetilde{\gamma})\|x-y\|+\gamma \alpha\|x-y\| \\
& =(1-(\widetilde{\gamma}-\gamma \alpha))\|x-y\| .
\end{aligned}
$$

for all $x, y \in H$. Therefore, $P_{F(T) \cap E P(\phi)}(I-A+\gamma f)$ is also a contraction, By the Banach theorem, there exists a unique element $x^{*} \in C$ such that

$$
x^{*}=P_{F(T) \cap E P(\phi)}(I-A+\gamma f)\left(x^{*}\right) .
$$

(II) Now we prove that the sequences $\left\{x_{n}\right\}$ and $\left\{u_{n}\right\}$ is bounded.

Let $p \in F(T) \cap E P(\phi)$. From the definition of $T_{r}$, we note that $u_{n}=T_{r_{n}} x_{n}$. It follows that

$$
\begin{aligned}
\left\|u_{n}-p\right\| & =\left\|T_{r_{n}} x_{n}-T_{r_{n}} p\right\| \\
& \leq\left\|x_{n}-p\right\| .
\end{aligned}
$$

From (3.1) and (3.2) we obtain

$$
\begin{aligned}
\left\|y_{n}-p\right\|^{2} & =\left\|P_{C}\left[\delta_{n} u_{n}+\left(1-\delta_{n}\right) T u_{n}\right]-p\right\|^{2} \\
& \leq\left\|\delta_{n}\left(u_{n}-p\right)+\left(1-\delta_{n}\right)\left(T u_{n}-p\right)\right\|^{2} \\
& =\delta_{n}\left\|u_{n}-p\right\|^{2}+\left(1-\delta_{n}\right)\left\|T u_{n}-p\right\|^{2}-\delta_{n}\left(1-\delta_{n}\right)\left\|T u_{n}-u_{n}\right\|^{2} \\
& \leq\left\|u_{n}-p\right\|^{2}-\left(1-\delta_{n}\right)\left(\delta_{n}-k\right)\left\|T u_{n}-u_{n}\right\|^{2} \\
& \leq\left\|u_{n}-p\right\|^{2} \leq\left\|x_{n}-p\right\|^{2} .
\end{aligned}
$$


Hence from (3.1) and (3.3) we have

$$
\begin{aligned}
\left\|x_{n+1}-p\right\| \leq & \left\|\alpha_{n}\left(\gamma f\left(x_{n}\right)-A p\right)+\beta_{n}\left(x_{n}-p\right)+\left(\left(1-\beta_{n}\right) I-\alpha_{n} A\right)\left(y_{n}-p\right)\right\| \\
\leq & \left(1-\beta_{n}-\alpha_{n} \widetilde{\gamma}\right)\left\|y_{n}-p\right\|+\beta_{n}\left\|x_{n}-p\right\|+\alpha_{n}\left\|\gamma f\left(x_{n}\right)-p\right\| \\
\leq & \left(1-\beta_{n}-\alpha_{n} \widetilde{\gamma}\right)\left\|x_{n}-p\right\|+\beta_{n}\left\|x_{n}-p\right\| \\
& +\alpha_{n} \gamma\left\|f\left(x_{n}\right)-f(p)\right\|+\alpha_{n}\|\gamma f(p)-A p\| \\
\leq & \left(1-\alpha_{n} \widetilde{\gamma}\right)\left\|x_{n}-p\right\|+\alpha_{n} \gamma \alpha\left\|x_{n}-p\right\|+\alpha_{n}\|\gamma f(p)-A p\| \\
\leq & \left(1-(\widetilde{\gamma}-\gamma \alpha) \alpha_{n}\right)\left\|x_{n}-p\right\|+\alpha_{n}\|\gamma f(p)-A p\| \\
\leq & \max \left\{\left\|x_{n}-p\right\|, \frac{1}{\widetilde{\gamma}-\gamma \alpha}\|\gamma f(p)-A p\|\right\} \\
\leq & \cdots \\
\leq & \max \left\{\left\|x_{1}-p\right\|, \frac{1}{\widetilde{\gamma}-\gamma \alpha}\|\gamma f(p)-A p\|\right\}, \quad \forall n \geq 1 .
\end{aligned}
$$

This implies that $\left\{x_{n}\right\}$ is a bounded sequence in $H$, and so $\left\{u_{n}\right\},\left\{T u_{n}\right\},\left\{y_{n}\right\}$, $\left\{A y_{n}\right\}$ and $\left\{f\left(x_{n}\right)\right\}$ are bounded sequences in $H$.

(III) Next we prove that $\left\|x_{n+1}-x_{n}\right\| \rightarrow 0$.

In fact, from $u_{n}=T_{r_{n}} x_{n}$ and $u_{n+1}=T_{r_{n+1}} x_{n+1}$, we have

$$
\phi\left(u_{n}, y\right)+\frac{1}{r_{n}}\left\langle y-u_{n}, u_{n}-x_{n}\right\rangle \geq 0, \quad \forall y \in C,
$$

and

$$
\phi\left(u_{n+1}, y\right)+\frac{1}{r_{n+1}}\left\langle y-u_{n+1}, u_{n+1}-x_{n+1}\right\rangle \geq 0, \forall y \in C,
$$

Putting $y=u_{n+1}$ in (3.4) and $y=u_{n}$ in (3.5), we have

$$
\phi\left(u_{n}, u_{n+1}\right)+\frac{1}{r_{n}}\left\langle u_{n+1}-u_{n}, u_{n}-x_{n}\right\rangle \geq 0,
$$

and

$$
\phi\left(u_{n+1}, u_{n}\right)+\frac{1}{r_{n+1}}\left\langle u_{n}-u_{n+1}, u_{n+1}-x_{n+1}\right\rangle \geq 0 .
$$

It follows from (A2) that

$$
\left\langle u_{n+1}-u_{n}, \frac{u_{n}-x_{n}}{r_{n}}-\frac{u_{n+1}-x_{n+1}}{r_{n+1}}\right\rangle \geq 0 .
$$

That is

$$
\left\langle u_{n+1}-u_{n}, u_{n}-u_{n+1}+u_{n+1}-x_{n}-\frac{r_{n}}{r_{n+1}}\left(u_{n+1}-x_{n+1}\right)\right\rangle \geq 0 .
$$

This implies that

$$
\begin{aligned}
\left\|u_{n+1}-u_{n}\right\|^{2} & \leq\left\langle u_{n+1}-u_{n}, x_{n+1}-x_{n}+\left(1-\frac{r_{n}}{r_{n+1}}\right)\left(u_{n+1}-x_{n+1}\right)\right\rangle \\
& \leq\left\|u_{n+1}-u_{n}\right\|\left\{\left\|x_{n+1}-x_{n}\right\|+\left|1-\frac{r_{n}}{r_{n+1}}\right| \cdot\left\|u_{n+1}-x_{n+1}\right\|\right\} .
\end{aligned}
$$


Since $\liminf \operatorname{in}_{n \rightarrow \infty} r_{n}>0$, without loss of generality, we may assume that there exists a real number $h$ such that $r_{n}>h>0$, for all $n \geq 1$. Then, we have

$$
\begin{aligned}
\left\|u_{n+1}-u_{n}\right\| & \leq\left\|x_{n+1}-x_{n}\right\|+\left|1-\frac{r_{n}}{r_{n+1}}\right| \cdot|| u_{n+1}-x_{n+1} \| \\
& \leq\left\|x_{n+1}-x_{n}\right\|+\frac{M}{h}\left|r_{n+1}-r_{n}\right| .
\end{aligned}
$$

where $M=\sup _{n>1}\left\{\left\|u_{n}-x_{n}\right\|\right\}$.

Define a mapping $T_{n} x:=\delta_{n} x+\left(1-\delta_{n}\right) T x$ for each $x \in C$. Then $T_{n}: C \rightarrow H$ is nonexpansive. Indeed,by using (1.2), (3.1), Lemma 2.1 and condition (ii), we have for all $x, y \in C$ that

$$
\begin{aligned}
\left\|T_{n} x-T_{n} y\right\|^{2} \\
\leq \\
=\delta_{n}\left\|x-y \delta_{n}(x-y)+\left(1-\delta_{n}\right)(T x-T y)\right\|^{2} \\
\leq \delta_{n}\|x-y\|^{2}+\left(1-\delta_{n}\right)\|T x-T y\|^{2}-\delta_{n}\left(1-\delta_{n}\right)\|x-T x-(y-T y)\|^{2} \\
\quad-\delta_{n}\left(1-\delta_{n}\right)\|x-T x-(y-T y)\|^{2} \\
=\|x-y\|^{2}-\left(1-\delta_{n}\right)\left(\delta_{n}-k\right)\|x-T x-(y-T y)\|^{2} \\
\leq\|x-y\|^{2},
\end{aligned}
$$

which implies that $T_{n}: C \rightarrow H$ is nonexpansive.

By using (3.1) and noting that $T_{n}$ is nonexpansive, we have

$$
\begin{aligned}
\left\|y_{n+1}-y_{n}\right\|= & \left\|T_{n+1} u_{n+1}-T_{n} u_{n}\right\| \\
= & \left\|T_{n+1} u_{n+1}-T_{n+1} u_{n}+T_{n+1} u_{n}-T_{n} u_{n}\right\| \\
\leq & \left\|u_{n+1}-u_{n}\right\|+\left\|T_{n+1} u_{n}-T_{n} u_{n}\right\| \\
= & \left\|u_{n+1}-u_{n}\right\|+\| \delta_{n+1} u_{n}+\left(1-\delta_{n+1}\right) T u_{n} \\
& -\left(\delta_{n} u_{n}+\left(1-\delta_{n}\right) T u_{n}\right) \| \\
\leq & \left\|u_{n+1}-u_{n}\right\|+\left|\delta_{n+1}-\delta_{n}\right|\left\|u_{n}-T u_{n}\right\| \\
\leq & \left\|u_{n+1}-u_{n}\right\|+M_{1}\left|\delta_{n+1}-\delta_{n}\right|,
\end{aligned}
$$

where $M_{1}=\sup _{n>1}\left\{\left\|u_{n}-T u_{n}\right\|\right\}$.

Letting $x_{n+1}=\left(1-\beta_{n}\right) z_{n}+\beta_{n} x_{n}, n \geq 1$. Then we have

$$
\begin{aligned}
z_{n+1}-z_{n}= & \frac{x_{n+2}-\beta_{n+1} x_{n+1}}{1-\beta_{n+1}}-\frac{x_{n+1}-\beta_{n} x_{n}}{1-\beta_{n}} \\
= & \frac{\alpha_{n+1} \gamma f\left(x_{n+1}\right)+\left(\left(1-\beta_{n+1}\right) I-\alpha_{n+1} A\right) y_{n+1}}{1-\beta_{n+1}} \\
& -\frac{\alpha_{n} \gamma f\left(x_{n}\right)+\left(\left(1-\beta_{n}\right) I-\alpha_{n} A\right) y_{n}}{1-\beta_{n}} \\
= & \frac{\alpha_{n+1}}{1-\beta_{n+1}}\left(\gamma f\left(x_{n+1}\right)-A y_{n+1}\right) \\
& +\frac{\alpha_{n}}{1-\beta_{n}}\left(A y_{n}-\gamma f\left(x_{n}\right)\right)+y_{n+1}-y_{n} .
\end{aligned}
$$


From (3.6) and (3.7), we get

$$
\begin{aligned}
& \left\|z_{n+1}-z_{n}\right\|-\left\|x_{n+1}-x_{n}\right\| \\
& \leq \frac{\alpha_{n+1}}{1-\beta_{n+1}}\left(\left\|\gamma f\left(x_{n+1}\right)\right\|+\left\|A y_{n+1}\right\|\right)+\frac{\alpha_{n}}{1-\beta_{n}}\left(\left\|A y_{n}\right\|+\left\|\gamma f\left(x_{n}\right)\right\|\right) \\
& \quad+\left\|y_{n+1}-y_{n}\right\|-\left\|x_{n+1}-x_{n}\right\| \\
& \leq \frac{\alpha_{n+1}}{1-\beta_{n+1}}\left(\left\|\gamma f\left(x_{n+1}\right)\right\|+\left\|A y_{n+1}\right\|\right)+\frac{\alpha_{n}}{1-\beta_{n}}\left(\left\|A y_{n}\right\|+\left\|\gamma f\left(x_{n}\right)\right\|\right) \\
& \quad+\left\|u_{n+1}-u_{n}\right\|+M_{1}\left|\delta_{n+1}-\delta_{n}\right|-\left\|x_{n+1}-x_{n}\right\| \\
& \leq \frac{\alpha_{n+1}}{1-\beta_{n+1}}\left(\left\|\gamma f\left(x_{n+1}\right)\right\|+\left\|A y_{n+1}\right\|\right)+\frac{\alpha_{n}}{1-\beta_{n}}\left(\left\|A y_{n}\right\|+\left\|\gamma f\left(x_{n}\right)\right\|\right) \\
& \quad+M_{1}\left|\delta_{n+1}-\delta_{n}\right|+\frac{M}{h}\left|r_{n+1}-r_{n}\right| .
\end{aligned}
$$

By conditions (i)-(iv) and $\left\{A y_{n}\right\},\left\{f\left(x_{n}\right)\right\}$ are bounded, we have

$$
\limsup _{n \rightarrow \infty}\left(\left\|z_{n+1}-z_{n}\right\|-\left\|x_{n+1}-x_{n}\right\|\right) \leq 0 .
$$

Hence by Lemma 2.2 we have

$$
\lim _{n \rightarrow \infty}\left\|z_{n}-x_{n}\right\|=0 .
$$

Consequently

$$
\lim _{n \rightarrow \infty}\left\|x_{n+1}-x_{n}\right\|=\lim _{n \rightarrow \infty}\left(1-\beta_{n}\right)\left\|z_{n}-x_{n}\right\|=0 .
$$

Hence from (3.1), we can obtain

$$
\begin{aligned}
\left\|x_{n}-y_{n}\right\| & \leq\left\|x_{n}-x_{n+1}\right\|+\left\|x_{n+1}-y_{n}\right\| \\
& \leq\left\|x_{n}-x_{n+1}\right\|+\left\|\alpha_{n}\left(\gamma f\left(x_{n}\right)-A y_{n}\right)+\beta_{n}\left(x_{n}-y_{n}\right)\right\| \\
& \leq\left\|x_{n}-x_{n+1}\right\|+\alpha_{n}\left(\left\|\gamma f\left(x_{n}\right)\right\|+\left\|A y_{n}\right\|\right)+\beta_{n}\left\|x_{n}-y_{n}\right\|,
\end{aligned}
$$

that is

$$
\left\|x_{n}-y_{n}\right\| \leq \frac{1}{1-\beta_{n}}\left\|x_{n}-x_{n+1}\right\|+\frac{\alpha_{n}}{1-\beta_{n}}\left(\left\|\gamma f\left(x_{n}\right)\right\|+\left\|A y_{n}\right\|\right),
$$

which together with condition (i),(iii) and (3.8) implies

$$
\lim _{n \rightarrow \infty}\left\|x_{n}-y_{n}\right\|=0 .
$$

(IV) Next we prove that $\left\|x_{n}-u_{n}\right\| \rightarrow 0$.

Indeed, for any given $z \in F(T) \cap E P(\phi)$, since $T_{r}$ is firmly nonexpansive, then we have

$$
\begin{aligned}
\left\|u_{n}-z\right\|^{2} & =\left\|T_{r_{n}} x_{n}-T_{r_{n}} z\right\|^{2} \\
& \leq\left\langle T_{r_{n}} x_{n}-T_{r_{n}} z, x_{n}-z\right\rangle \\
& =\left\langle u_{n}-z, x_{n}-z\right\rangle \\
& =\frac{1}{2}\left(\left\|u_{n}-z\right\|^{2}+\left\|x_{n}-z\right\|^{2}-\left\|x_{n}-u_{n}\right\|^{2}\right) .
\end{aligned}
$$

It follows that

$$
\left\|u_{n}-z\right\|^{2} \leq\left\|x_{n}-z\right\|^{2}-\left\|x_{n}-u_{n}\right\|^{2}
$$


Using Lemma2.6, (3.1), (3.3) and (3.10), we have

$$
\begin{aligned}
&\left\|x_{n+1}-z\right\|^{2} \\
&=\left\|\alpha_{n}\left(\gamma f\left(x_{n}\right)-A z\right)+\beta_{n}\left(x_{n}-y_{n}\right)+\left(I-\alpha_{n} A\right)\left(y_{n}-z\right)\right\|^{2} \\
& \leq\left\|\left(I-\alpha_{n} A\right)\left(y_{n}-z\right)+\beta_{n}\left(x_{n}-y_{n}\right)\right\|^{2}+2 \alpha_{n}\left\langle\gamma f\left(x_{n}\right)-A z, x_{n+1}-z\right\rangle \\
& \leq {\left[\left\|I-\alpha_{n} A\right\|\left\|y_{n}-z\right\|+\beta_{n}\left\|x_{n}-y_{n}\right\|\right]^{2}+2 \alpha_{n}\left\|\gamma f\left(x_{n}\right)-A z\right\|\left\|x_{n+1}-z\right\| } \\
& \leq {\left[\left(1-\alpha_{n} \widetilde{\gamma}\right)\left\|u_{n}-z\right\|+\beta_{n}\left\|x_{n}-y_{n}\right\|\right]^{2}+2 \alpha_{n}\left\|\gamma f\left(x_{n}\right)-A z\right\|\left\|x_{n+1}-z\right\| } \\
& \leq\left(1-\alpha_{n} \widetilde{\gamma}\right)^{2}\left\|u_{n}-z\right\|^{2}+2\left(1-\alpha_{n} \widetilde{\gamma}\right) \beta_{n}\left\|u_{n}-z\right\|\left\|x_{n}-y_{n}\right\| \\
&+\beta_{n}^{2}\left\|x_{n}-y_{n}\right\|^{2}+2 \alpha_{n}\left\|\gamma f\left(x_{n}\right)-A z\right\|\left\|x_{n+1}-z\right\| \\
& \leq\left(1-\alpha_{n} \widetilde{\gamma}\right)^{2}\left[\left\|x_{n}-z\right\|^{2}-\left\|x_{n}-u_{n}\right\|^{2}\right]+2\left(1-\alpha_{n} \widetilde{\gamma}\right) \beta_{n}\left\|u_{n}-z\right\|\left\|x_{n}-y_{n}\right\| \\
&+\beta_{n}^{2}\left\|x_{n}-y_{n}\right\|^{2}+2 \alpha_{n}\left\|\gamma f\left(x_{n}\right)-A z\right\|\left\|x_{n+1}-z\right\| \\
& \leq\left(1-2 \alpha_{n} \widetilde{\gamma}+\left(\alpha_{n} \widetilde{\gamma}\right)^{2}\right)\left\|x_{n}-z\right\|^{2}-\left(1-\alpha_{n} \widetilde{\gamma}\right)^{2}\left\|x_{n}-u_{n}\right\|\left\|^{2}+\beta_{n}^{2}\right\| x_{n}-y_{n} \|^{2} \\
&+2\left(1-\alpha_{n} \widetilde{\gamma}\right) \beta_{n}\left\|u_{n}-z\right\|\left\|x_{n}-y_{n}\right\|+2 \alpha_{n}\left\|\gamma f\left(x_{n}\right)-A z\right\|\left\|x_{n+1}-z\right\| \\
& \leq\left\|x_{n}-z\right\|^{2}+\alpha_{n} \widetilde{\gamma}^{2}\left\|x_{n}-z\right\|^{2}-\left(1-\alpha_{n} \widetilde{\gamma}\right)^{2}\left\|x_{n}-u_{n}\right\|^{2}+\beta_{n}^{2}\left\|x_{n}-y_{n}\right\|^{2} \\
&+ 2\left(1-\alpha_{n} \widetilde{\gamma}\right) \beta_{n}\left\|u_{n}-z\right\|\left\|x_{n}-y_{n}\right\|+2 \alpha_{n}\left\|\gamma f\left(x_{n}\right)-A z\right\|\left\|x_{n+1}-z\right\| .
\end{aligned}
$$

Then we have

$$
\begin{aligned}
(1- & \left.\alpha_{n} \widetilde{\gamma}\right)^{2}\left\|x_{n}-u_{n}\right\|^{2} \\
\leq & \left\|x_{n}-z\right\|^{2}-\left\|x_{n+1}-z\right\|^{2}+\alpha_{n} \widetilde{\gamma}^{2}\left\|x_{n}-z\right\|^{2}+\beta_{n}^{2}\left\|x_{n}-y_{n}\right\|^{2} \\
& +2\left(1-\alpha_{n} \widetilde{\gamma}\right) \beta_{n}\left\|u_{n}-z\right\|\left\|x_{n}-y_{n}\right\|+2 \alpha_{n}\left\|\gamma f\left(x_{n}\right)-A z\right\|\left\|x_{n+1}-z\right\| \\
\leq & \left\|x_{n}-x_{n+1}\right\|\left(\left\|x_{n}-z\right\|+\left\|x_{n+1}-z\right\|\right)+\alpha_{n} \widetilde{\gamma}^{2}\left\|x_{n}-z\right\|^{2}+\beta_{n}^{2}\left\|x_{n}-y_{n}\right\|^{2} \\
& +2\left(1-\alpha_{n} \widetilde{\gamma}\right) \beta_{n}\left\|u_{n}-z\right\|\left\|x_{n}-y_{n}\right\|+2 \alpha_{n}\left\|\gamma f\left(x_{n}\right)-A z\right\|\left\|x_{n+1}-z\right\| .
\end{aligned}
$$

By virtue of comdition (i) $\alpha_{n} \rightarrow 0,(3.8)$ and (3.9), note that $\left\{f\left(x_{n}\right)\right\},\left\{x_{n}\right\},\left\{u_{n}\right\}$ are bounded, these imply that

$$
\left\|x_{n}-u_{n}\right\| \rightarrow 0, \quad(\text { as } \quad n \rightarrow \infty) .
$$

(V) Next we prove that $\left\|u_{n}-S u_{n}\right\| \rightarrow 0$.

From condition (ii), we have $\delta_{n} \rightarrow \lambda$ as $n \rightarrow \infty$, where $\lambda \in[k, 1)$. Define $S: C \rightarrow H$ by $S x=\lambda x+(1-\lambda) T x$. Then, $S$ is nonexpansive with $F(S)=F(T)$ by Lemma 2.5. Notice that

$$
\begin{aligned}
\left\|x_{n}-S u_{n}\right\| & \leq\left\|x_{n}-y_{n}\right\|+\left\|y_{n}-S u_{n}\right\| \\
& =\left\|x_{n}-y_{n}\right\|+\left\|\delta_{n} u_{n}+\left(1-\delta_{n}\right) T u_{n}-\left(\lambda u_{n}+(1-\lambda) T u_{n}\right)\right\| \\
& \left.\leq\left\|x_{n}-y_{n}\right\|+\mid \delta_{n}-\lambda\|\| u_{n}-T u_{n}\right] \|,
\end{aligned}
$$

which combines with (3.9) yielding that

$$
\lim _{n \rightarrow \infty}\left\|x_{n}-S u_{n}\right\|=0 .
$$

Observe that

$$
\left\|u_{n}-S u_{n}\right\| \leq\left\|u_{n}-x_{n}\right\|+\left\|x_{n}-S u_{n}\right\|
$$


From (3.11) and (3.12), we have

$$
\lim _{n \rightarrow \infty}\left\|u_{n}-S u_{n}\right\|=0 .
$$

(VI) Next we prove that

$$
\limsup _{n \rightarrow \infty}\left\langle(\gamma f-A) p, x_{n}-p\right\rangle \leq 0,
$$

where $p=P_{F(T) \cap E P(\phi)}(I-A+\gamma f)(p)$. To show this inequality, we can choose a subsequence $\left\{u_{n_{i}}\right\}$ of $\left\{u_{n}\right\}$ such that

$$
\limsup _{n \rightarrow \infty}\left\langle(\gamma f-A)(p), S u_{n}-p\right\rangle=\lim _{i \rightarrow \infty}\left\langle(\gamma f-A) p, S u_{n_{i}}-p\right\rangle .
$$

Since $\left\{u_{n_{i}}\right\}$ is bounded in $C$, without loss of generality, we can assume that $u_{n_{i}} \rightarrow$ $w \in C$ as $i \rightarrow \infty$. Now we prove that $w \in F(T) \cap E P(\phi)$. Now we show that $w \in E P(\phi)$. In fact, since $u_{n}=T_{r_{n}} x_{n}$, we have

$$
\phi\left(u_{n}, y\right)+\frac{1}{r_{n}}\left\langle y-u_{n}, u_{n}-x_{n}\right\rangle \geq 0, \quad \forall y \in C .
$$

By condition (A2), we have

$$
\frac{1}{r_{n}}\left\langle y-u_{n}, u_{n}-x_{n}\right\rangle \geq \phi\left(y, u_{n}\right)
$$

and hence

$$
\left\langle y-u_{n_{i}}, \frac{u_{n_{i}}-x_{n_{i}}}{r_{n_{i}}}\right\rangle \geq \phi\left(y, u_{n_{i}}\right) .
$$

Since $\left\|u_{n_{i}}-x_{n_{i}}\right\| \rightarrow 0$ and $u_{n_{i}} \rightarrow w$, from condition (A4), we have

$$
\phi(y, w) \leq 0, \quad \forall y \in C .
$$

For any $t \in(0,1]$ and $y \in C$, let $y_{t}=t y+(1-t) w$, then $y_{t} \in C$ and $\phi\left(y_{t}, w\right) \leq 0$. From (A1) and (A4), we have

$$
\begin{aligned}
0 & =\phi\left(y_{t}, y_{t}\right)=\phi\left(y_{t}, t y+(1-t) w\right) \\
& =t \phi\left(y_{t}, y\right)+(1-t) \phi\left(y_{t}, w\right) \\
& \leq t \phi\left(y_{t}, y\right),
\end{aligned}
$$

and hence

$$
\phi\left(y_{t}, y\right) \geq 0 \text {. }
$$

By condition (A3), we have $\phi(w, y) \geq 0, \forall y \in C$. Hence $w \in E P(\phi)$.

We shall show $w \in F(T)$. Since Hilbert spaces are Opial's spaces, suppose the contrary, $w \notin F(S)$, i.e., $w \neq S w$. Since $u_{n_{i}} \rightarrow w$, from Opial's condition and (3.13), we have

$$
\begin{aligned}
\liminf _{i \rightarrow \infty}\left\|u_{n_{i}}-w\right\| & <\liminf _{i \rightarrow \infty}\left\|u_{n_{i}}-S w\right\| \\
& \leq \liminf _{i \rightarrow \infty}\left(\left\|u_{n_{i}}-S u_{n_{i}}\right\|+\left\|S u_{n_{i}}-S w\right\|\right) \\
& \leq \liminf _{i \rightarrow \infty}\left\|S u_{n_{i}}-S w\right\| \\
& \leq \liminf _{i \rightarrow \infty}\left\|u_{n_{i}}-w\right\|
\end{aligned}
$$


This is a contradiction. We get $w \in F(S)$. Again by Lemma 2.5, we have $w \in F(S)=F(T)$. Therefore $w \in F(T) \cap E P(\phi)$.

Since $p=P_{F(T) \cap E P(\phi)}(I-A+\gamma f)(p)$. It follows from (3.12),(3.13),(3.15) and Remark 1 that

$$
\begin{aligned}
\limsup _{n \rightarrow \infty}\left\langle(\gamma f-A)(p), x_{n}-p\right\rangle & =\limsup _{n \rightarrow \infty}\left\langle(\gamma f-A)(p), x_{n}-S u_{n}+S u_{n}-p\right\rangle \\
& \leq \limsup _{n \rightarrow \infty}\left\langle(\gamma f-A)(p), S u_{n}-p\right\rangle \\
& =\lim _{i \rightarrow \infty}\left\langle(\gamma f-A)(p), S u_{n_{i}}-p\right\rangle \\
& =\lim _{i \rightarrow \infty}\left\langle(\gamma f-A)(p), S u_{n_{i}}-u_{n_{i}}+u_{n_{i}}-p\right\rangle \\
& =\langle(\gamma f-A)(p), w-p\rangle \leq 0 .
\end{aligned}
$$

(VII) Finally, we prove that $\left\{x_{n}\right\}$ and $\left\{u_{n}\right\}$ converge strongly to $p$. In fact, from (3.1),(3.3) and Lemma 2.6, we have

$$
\begin{aligned}
&\left\|x_{n+1}-p\right\|^{2} \\
&=\left\|\alpha_{n}\left(\gamma f\left(x_{n}\right)-A p\right)+\beta_{n}\left(x_{n}-p\right)+\left(\left(1-\beta_{n}\right) I-\alpha_{n} A\right)\left(y_{n}-p\right)\right\|^{2} \\
& \leq\left\|\left(\left(1-\beta_{n}\right) I-\alpha_{n} A\right)\left(y_{n}-p\right)+\beta_{n}\left(x_{n}-p\right)\right\|^{2}+2 \alpha_{n}\left\langle\gamma f\left(x_{n}\right)-A p, x_{n+1}-p\right\rangle \\
& \leq {\left[\left\|\left(\left(1-\beta_{n}\right) I-\alpha_{n} A\right)\left(y_{n}-p\right)\right\|+\left\|\beta_{n}\left(x_{n}-p\right)\right\|\right]^{2} } \\
&+2 \alpha_{n} \gamma\left\langle f\left(x_{n}\right)-f(p), x_{n+1}-p\right\rangle+2 \alpha_{n}\left\langle\gamma f(p)-A p, x_{n+1}-p\right\rangle \\
& \leq {\left[\left(1-\beta_{n}-\alpha_{n} \widetilde{\gamma}\right)\left\|y_{n}-p\right\|+\beta_{n}\left\|x_{n}-p\right\|\right]^{2}+2 \alpha_{n} \gamma \alpha\left\|x_{n}-p\right\|\left\|x_{n+1}-p\right\| } \\
&+2 \alpha_{n}\left\langle\gamma f(p)-A p, x_{n+1}-p\right\rangle \\
& \leq {\left[\left(1-\beta_{n}-\alpha_{n} \widetilde{\gamma}\right)\left\|x_{n}-p\right\|+\beta_{n}\left\|x_{n}-p\right\|\right]^{2}+2 \alpha_{n} \gamma \alpha\left\|x_{n}-p\right\|\left\|x_{n+1}-p\right\| } \\
&+2 \alpha_{n}\left\langle\gamma f(p)-A p, x_{n+1}-p\right\rangle \\
& \leq\left(1-\alpha_{n} \widetilde{\gamma}\right)^{2}\left\|x_{n}-p\right\|^{2}+\alpha_{n} \gamma \alpha\left\{\left\|x_{n}-p\right\|^{2}+\left\|x_{n+1}-p\right\|^{2}\right\} \\
&+2 \alpha_{n}\left\langle\gamma f(p)-A p, x_{n+1}-p\right\rangle,
\end{aligned}
$$

which implies that

$$
\begin{aligned}
\left\|x_{n+1}-p\right\|^{2} \leq & \frac{\left(1-\alpha_{n} \widetilde{\gamma}\right)^{2}+\alpha_{n} \gamma \alpha}{1-\alpha_{n} \gamma \alpha}\left\|x_{n}-p\right\|^{2}+\frac{2 \alpha_{n}}{1-\alpha_{n} \gamma \alpha}\left\langle f(p)-p, x_{n+1}-p\right\rangle \\
\leq & \left(1-\frac{2 \alpha_{n}(\widetilde{\gamma}-\gamma \alpha)}{1-\alpha_{n} \gamma \alpha}\right)\left\|x_{n}-p\right\|^{2}+\frac{\left(\alpha_{n} \widetilde{\gamma}\right)^{2}}{1-\alpha_{n} \gamma \alpha}\left\|x_{n}-p\right\|^{2} \\
& +\frac{2 \alpha_{n}}{1-\alpha_{n} \gamma \alpha}\left\langle f(p)-p, x_{n+1}-p\right\rangle \\
\leq & \left(1-\frac{2 \alpha_{n}(\widetilde{\gamma}-\gamma \alpha)}{1-\alpha_{n} \gamma \alpha}\right)\left\|x_{n}-p\right\|^{2}+\frac{2 \alpha_{n}(\widetilde{\gamma}-\gamma \alpha)}{1-\alpha_{n} \gamma \alpha} \\
& \times\left(\frac{\alpha_{n} \widetilde{\gamma}^{2} M_{2}}{2(\widetilde{\gamma}-\gamma \alpha)}+\frac{1}{\widetilde{\gamma}-\gamma \alpha}\left\langle f(p)-p, x_{n+1}-p\right\rangle\right) \\
= & \left(1-\varrho_{n}\right)\left\|x_{n}-p\right\|^{2}+\varrho_{n} \sigma_{n},
\end{aligned}
$$


where $M_{2}=\sup _{n \geq 1}\left\{\left\|x_{n}-p\right\|^{2}\right\}$,

$$
\varrho_{n}=\frac{2 \alpha_{n}(\widetilde{\gamma}-\gamma \alpha)}{1-\alpha_{n} \gamma \alpha} \quad \text { and } \quad \sigma_{n}=\frac{\alpha_{n} \widetilde{\gamma}^{2} M_{2}}{2(\widetilde{\gamma}-\gamma \alpha)}+\frac{1}{\widetilde{\gamma}-\gamma \alpha}\left\langle f(p)-p, x_{n+1}-p\right\rangle .
$$

From condition (i) and (3.14) that $\varrho_{n} \rightarrow 0, \sum_{n=1}^{\infty} \varrho_{n}=\infty$ and $\lim \sup _{n \rightarrow \infty} \sigma_{n} \leq 0$. Hence, by Lemma 2.3, the sequence $\left\{x_{n}\right\}$ converges strongly to $p$. Consequently, we can obtain that $\left\{u_{n}\right\}$ also converges strongly to $p$.

Taking $\gamma=1$ and $A=I$ is an identity mapping in Theorem 3.1, we can obtain the following results immediately.

Theorem 3.2. Let $H$ be a real Hilbert space, $C$ be a nonempty closed convex subset of $H$. let $\phi: C \times C \rightarrow \mathbb{R}$ be a bifunction satisfying $(A 1)-(A 4)$. Let $T: C \rightarrow H$ be a $k$-strictly pseudo-contractive nonself-mapping such that $F(T) \cap$ $E P(\phi) \neq \emptyset$, and $f: H \rightarrow H$ be a contractive mapping with a contractive constant $\alpha \in(0,1)$. For any given $x_{1} \in H$, let $\left\{x_{n}\right\}$ and $\left\{u_{n}\right\}$ be the iterative sequence defined by

$$
\begin{cases}\phi\left(u_{n}, y\right)+\frac{1}{r_{n}}\left\langle y-u_{n}, u_{n}-x_{n}\right\rangle \geq 0, & \forall y \in C, \\ y_{n}=\delta_{n} u_{n}+\left(1-\delta_{n}\right) T u_{n}, & \\ x_{n+1}=\alpha_{n} f\left(x_{n}\right)+\beta_{n} x_{n}+\left(1-\beta_{n}-\alpha_{n}\right) y_{n}, & \forall n \geq 1 .\end{cases}
$$

where $\left\{\alpha_{n}\right\},\left\{\beta_{n}\right\},\left\{\delta_{n}\right\}$ are three sequences in $[0,1]$ and $r_{n} \subset(0, \infty)$. If the following conditions are satisfied:

(i) $\lim _{n \rightarrow \infty} \alpha_{n}=0 ; \quad \sum_{n=1}^{\infty} \alpha_{n}=\infty$,

(ii) $k \leq \delta_{n} \leq \lambda<1$ for all $n \geq 1$ and $\sum_{n=1}^{\infty}\left|\delta_{n+1}-\delta_{n}\right|<\infty$,

(iii) $0<\liminf _{n \rightarrow \infty} \beta_{n} \leq \limsup _{n \rightarrow \infty} \beta_{n}<1$,

(iv) $\liminf \operatorname{in}_{n \rightarrow \infty} r_{n}>0, \lim _{n \rightarrow \infty}\left|r_{n+1}-r_{n}\right|=0$.

Then $\left\{x_{n}\right\}$ and $\left\{u_{n}\right\}$ cnoverge strongly to $p \in F(T) \cap E P(\phi)$, where $p=P_{F(T) \cap E P(\phi)} f(p)$.

\section{REFERENCES}

1. E. Blum, W. Oettli, From optimization and variational inequalities to equilibrium problems, Math. Student. 63 (1994), 123-145.

2. F. E. Browder, Fixed point theorems for noncompact mappings in Hilbert spaces, Proc. Natl. Acad. Sci. USA, 53 (1965), 1272-1276.

3. F. E. Browder, W. V. Petryshyn, Construction of fixed points of nonlinear mappings in Hilbert spaces, J. Math. Anal. Appl. 20 (1967), 197-228.

4. L. C. Ceng, S.A.Homidan, Q.H.Ansari, J. C. Yao, An iterative scheme for equilibrium problems and fixed point problems of strict pseudo-contraction mappings, J. Comput. Appl. Math. 223 (2009), 967-974.

5. S. S. Chang, Some problems and results in the study of nonlinear analysis, Nonlinear Anal. 30 (1997), 4197-4208.

6. P. L. Combettes, A. Hirstoaga, Equilibrium programming in Hilbert spaces, J. Nonlinear Convex Anal. 6 (2005), 117-136. 
7. S. D. Flam, A. S. Antipin, Equilibrium programming using proximal-like algorithms, Math. Program. 78 (1997), 29-41.

8. F. Flores-Bazan, Existence theory for finite-dimensional pseudomonotone equilibrium problems, Acta. Appl. Math. 77 (2003), 249-297.

9. N. Hadjisavvas, S. Schaible, From scalar to vector equilibrium problems in the quasimonotone case, J. Optim. Theory Appl. 96 (1998), 297-309.

10. H. Iiduka, W. Takahashi , Strong convergence theorems for nonexpansive mappings and inverse-strongly monotone mappings, Nonlinear Anal. 61 (2005), 341-350.

11. T. H. Kim, H. K. Xu, Strong convergence of modified Mann iteration, Nonlinear Anal. 61 (2005), 51-60.

12. W. R. Mann, Mean value methods in iterations, Proc. Amer. Math. Soc. 4 (1953), 506-510.

13. G. Marino, H. K. Xu, Weak and strong convergence theorems for $k$-strict pseudocontractions in Hilbert spaces, J. Math. Anal. Appl. 329 (2007), 336-349.

14. A. Moudafi Viscosity approximation methods for fixed points problems, J. Math. Anal. Appl. 241 (2000), 46-55.

15. K. Nakajo, W. Takahashi, Strong convergence theorems for nonexpansive mappings and nonexpansive semigroups, J. Math. Anal. Appl. 279 (2003), 372-379.

16. Z. Opial, Weak convergence of the sequence of successive approximations for nonexpansive mappings, Bull. Amer. Math. Soc. 73 (1967), 595-597.

17. S. Reich, Weak convergence theorems for nonexpansive mappings in Banach spaces, J. Math. Anal. Appl. 67 (1979), 274-276.

18. B. E. Rhoades, Fixed point iterations using infinite matrices, Trans. Amer. Math. Soc. 196 (1974), 162-176.

19. T. Suzuki, Strong convergence of Krasnoselskii and Mann's type sequences for oneparameter nonexpansive semigroups without Bochner integrals, J. Math. Anal. Appl. 305 (2005), 227239.

20. A. Tada, W. Takahashi, Strong convergence theorem for an equilibrium problem and a nonexpansive mapping, in: W. Takahashi, T. Tanaka(Eds.), Nonlinear Analysis and Convex Analysis, 609-617, Yokohama Publishers, Yokohama, 2006.

21. S. Takahashi, W. Takahashi, Viscosity approximation methods for equilibrium problems and fixed point problems in Hilbert spaces, J. Math. Anal. Appl. 331 (2007), 506-515.

22. W. Takahashi, M. Toyoda, Weak convergence theorems for nonexpansive mappings and monotone mappings, J. Optim. Theory Appl. 118 (2003), 417-428.

23. R. Wittmann, Approximation of fixed points of nonexpansive mappings, Arch. Math. 58 (1992), 486-491.

24. H. K. Xu, Iterative algorithms for nonlinear operators, J. London Math. Soc. 66 (2002), 240-256.

25. H.Zhou, Convergence theorems of fixed Points for $k$-strict pseudo-contractions in Hilbert spaces, Nonlinear Anal. 69 (2008), 456-462.

1 Department of Mathematics, Yibin University, Yibin, Sichuan 644000, China

E-mail address: zhaolcyb@yahoo.com.cn

2 Department of Mathematics, Sichuan University, Chengdu 610064, P. R. China

E-mail address: changss@yahoo.cn 